\title{
e-VLBI related activities at Onsala Space Observatory
}

\author{
Simon Casey ${ }^{1}$ \\ Onsala Space Observatory \\ SE-439 92 Onsala, SWEDEN \\ E-mail: simon. caseyechalmers.se
}

\section{Rüdiger Haas}

Onsala Space Observatory

SE-439 92 Onsala, SWEDEN

E-mail: rudiger. haas lchalmers.se

\section{Michael Lindqvist}

Onsala Space Observatory

SE-439 92 Onsala, SWEDEN

E-mail: michael. Iindqvistechalmers.se

\section{Roger Hammargren}

Onsala Space Observatory

SE-439 92 Onsala, SWEDEN

E-mail: roger. hammargren@chalmers. se

\section{John Conway}

Onsala Space Observatory

SE-439 92 Onsala, SWEDEN

E-mail: john. conwaylchalmers.se

\section{Jan Johansson}

Onsala Space Observatory

SE-439 92 Onsala, SWEDEN

E-mail: jan.johanssonechalmers.se

This paper details e-VLBI related work performed at Onsala Space Observatory for Astronomy and Geodesy. The $10 \mathrm{Gbit} / \mathrm{s}$ network connectivity is discussed, and results from $4 \mathrm{Gbit} / \mathrm{s}$ spectral line observations as well as Fennoscandian ultra-rapid dUT1 experiments are presented.

Science and Technology of Long Baseline Real-Time Interferometry:

The 8th International e-VLBI Workshop - EXPReS09

Madrid, Spain

June 22-26, 2009

\footnotetext{
1 Speaker
} 


\section{Introduction}

Onsala Space Observatory has been actively involved in VLBI since the 1960s with tapebased observations, and helped to produce the first transatlantic VLBI fringes between Onsala and Westford, Massachusetts in 1968. Less than 40 years later, in March 2004, the first realtime transatlantic e-VLBI fringes were achieved, again between the same pair of antennas.

\section{Network connections}

In November 2003, Onsala Space Observatory was linked to the Swedish academic Internet backbone provided by SUNET, by way of a pair of Gigabit Ethernet (GE) links multiplexed over a single optical fibre pair. One link was used for general internet access at the observatory, whilst the other was reserved for e-VLBI transfers. This GE enabled real-time eVLBI transfers in the EVN at rates of up to $512 \mathrm{Mbit} / \mathrm{s}$. As part of the EXPReS project, the connectivity was enhanced through the installation of an additional $10 \mathrm{GE}$ link in order to accommodate true $1 \mathrm{Gbit} / \mathrm{s}$ e-VLBI and other future projects in November 2007. The existing Coarse Wavelength Division Multiplexing (CWDM) equipment was replaced by Dense Wavelength Division Multiplexing (DWDM) equipment, which allowed both the original 2x 1 GE links to continue operating as before whilst adding a $10 \mathrm{GE}$ link over the same fibre pair.

At the observatory, the $10 \mathrm{GE}$ link is presented as a long reach fibre optic link and connects to an HP 6400cl 6-port CX-4 switch. The switch provides 6x 10 gigabit CX-4 ports at the front, and a pair of X2 modular ports at the rear, one of which is populated with a $10 \mathrm{GE}$ Long-Reach optic. The link runs as a dedicated lightpath between the observatory and a NORDUnet point of presence (POP) located in Stockholm, where it terminates in a Juniper MX480 router (SUNET project router in Figure 1). From this router, a routed 10 gigabit connection is available to the IP network, as well as a 10 gigabit layer 2 connection to the Alcatel Transport Service Switch (TSS) platform implemented by NORDUnet. From the TSS, layer 2 connections can be made to several destinations; currently $4 \mathrm{Gbit} / \mathrm{s}$ is allocated within the TSS to Jodrell Bank, $1.5 \mathrm{Gbit} / \mathrm{s}$ to JIVE, and $4.5 \mathrm{Gbit} / \mathrm{s}$ to LOFAR.

Data streams are directed towards a particular destination through a combination of Virtual Local Area Network (VLAN) tags (ID numbers) and IP addressing. To reach the routed IP network, or JIVE via the TSS, devices based at the observatory are assigned an IP address from a routable $/ 29$ subnet allocated by SUNET. Ethernet frames from these devices are then given a VLAN tag at the HP switch, which informs the SUNET router that these frames should be routed to the IP network. At the same time, the SUNET router examines the destination IP address, and if this is contained within the JIVE IP address space, then the frames are instead tagged with a new VLAN ID and switched into the TSS platform and onto JIVE. In this respect, the link to JIVE is a routed connection over dedicated layer 2 links. Data streams intended for any other destination within the TSS are tagged with the relevant VLAN ID at the HP switch and transparently switched into the TSS by the SUNET router.

To communicate between Onsala and Metsähovi, data is routed over the shared Nordic academic IP network infrastructure, as seen in Figure 1, at rates of up to $10 \mathrm{Gbit} / \mathrm{s}$, dependent upon background traffic which is typically below $3 \mathrm{Gbit} / \mathrm{s}$. 


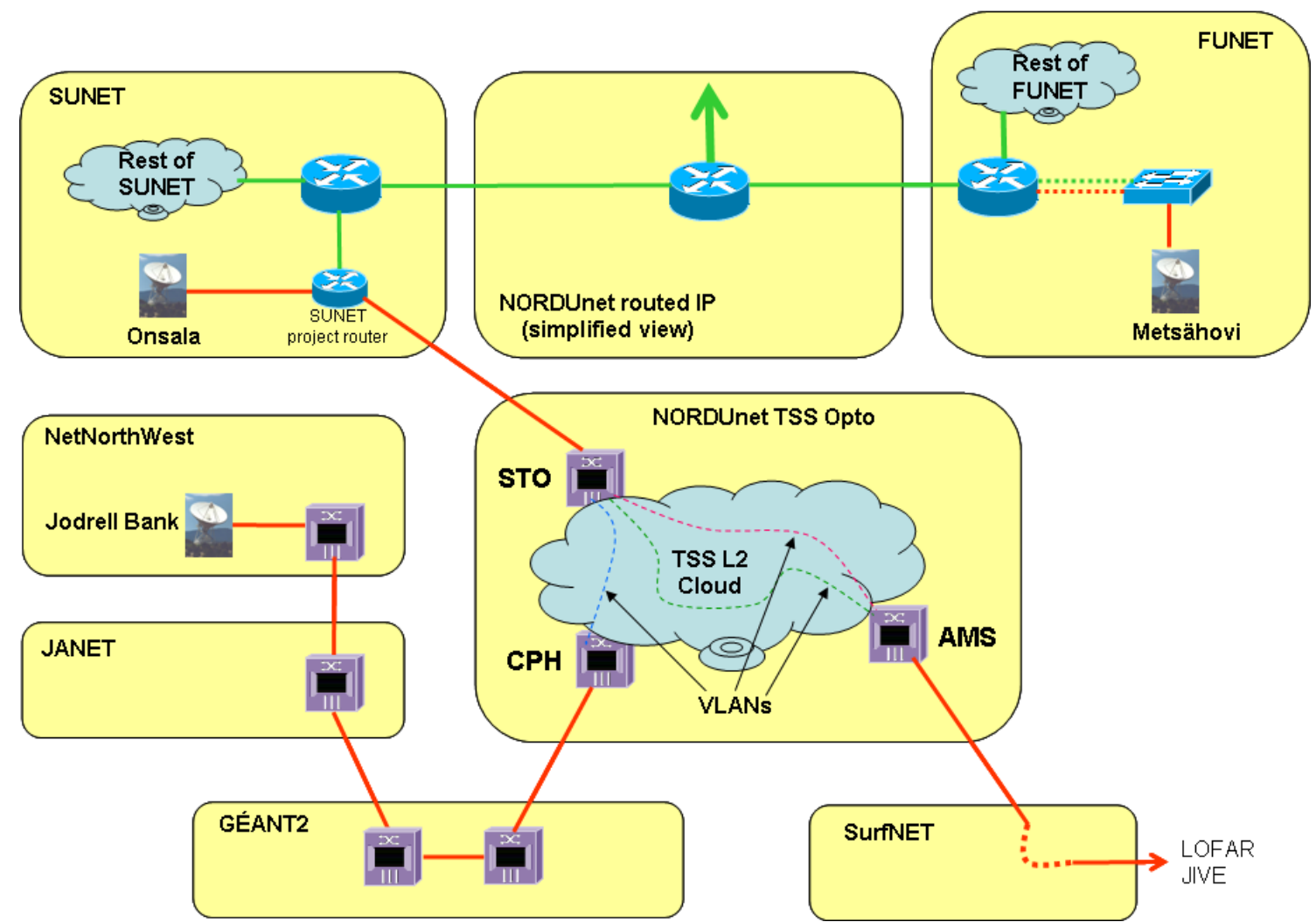

Figure 1: Network connectivity between Onsala and Metsähovi, LOFAR, JIVE, and Jodrell Bank.

\section{Astronomical work}

\subsection{VLBI observations}

In order to take advantage of the 10 gigabit link in e-VLBI observations, it was necessary to upgrade the motherboard of the Mark 5 data recorder and add a PCI-Express 10 GE Network Interface Card (NIC). This work was undertaken in the summer of 2008, and several network and VLBI formatter tests were performed at $1024 \mathrm{Mbit} / \mathrm{s}$ together with JIVE in the autumn of 2008. As a result, the third epoch observation of a flare in microquasar SS 433 [1] was successfully observed by the e-EVN on 19 November 2008 with antennas at Onsala, Effelsberg and Westerbork transmitting a full $1024 \mathrm{Mbit} / \mathrm{s}$ of e-VLBI data. Since then, Onsala has been participating regularly in e-VLBI experiments at $1024 \mathrm{Mbit} / \mathrm{s}$, as well as being involved in demonstrations for the International Year of Astronomy.

\section{2 iBOB observations}

As part of the FABRIC Joint Research Activity within EXPReS, work has been undertaken at Onsala Space Observatory, Metsähovi Radio Observatory and Jodrell Bank Observatory towards the goal of connecting an external antenna into the UK-based e-MERLIN radio interferometer at a data rate of $4 \mathrm{Gbit} / \mathrm{s}$. For this purpose, data capture, transmission and reception, is performed by FPGA-based systems known as iBOBs (internet break-out boards) 
designed at UC Berkeley, which are programmed with sets of firmware written at Metsähovi and Jodrell Bank.


Figure 2: Water spectra of $W 3$ as captured by an $i B O B, 13 \mathrm{kHz}$ channel bandwidth, 30s integration. First plot shows on-source data over IF frequency range 256-512 MHz, second plot shows off-source data subtracted from on-source; third and fourth plots are as first and second but over IF frequency range 335-345 $\mathrm{MHz}$.

Leading up to correlation in the e-MERLIN correlator, various tests have been performed between Onsala and Metsähovi in order to demonstrate the ability of the iBOB to correctly sample and transmit radio astronomy data. One such test was conducted at the beginning of November 2008, with data captured from the 20m Onsala telescope being recorded in real-time on a PC based at Metsähovi. The radio maser W3 was observed at a frequency of $22 \mathrm{GHz}$, and the sampled data were successfully transmitted and received at a rate of $4 \mathrm{Gbit} / \mathrm{s}$ over a period of 5 minutes. For noise calibration purposes, a further 5 minutes were recorded whilst the telescope was not pointing at a radio source. Processing of the two data sets was performed at Onsala, and the results can be seen in Figure 2.

The e-MERLIN correlator is in the process of being commissioned, and work is ongoing to obtain fringes between Onsala and other e-MERLIN antennas.

\section{4. e-VLBI for geodesy}

Since 2005, e-VLBI has been used at Onsala for geodetic VLBI. The first one hour sessions on one baseline were performed with Tsukuba in July and August 2005. These sessions used offline e-transfer of the data from Onsala to Tsukuba. In September and November of 2005 we participated in the iGRID2005 and SuperComputing2005 conferences, and data were sent 
successfully in real-time from Onsala to Haystack and correlated successfully in real-time. The data rate was $512 \mathrm{Mbps}$ and fringes were achieved for several hours.

During the second half of 2006 we started to do e-VLBI tests with the Bonn correlator. The first successful offline e-transfer to Bonn was performed in November 2006. In December of 2006 we used the PC based VLBI data recorder, PC-EVN ${ }^{1}$, for the first time for geodesy. Parts of a standard R1-session were recorded on the PC-EVN and later sent offline to Bonn with the Tsunami-protocol.

In 2007 we started together with our colleagues in Metsähovi, Kashima and Tsukuba, a project for Fennoscandian-Japanese ultra-rapid dUT1 (UT1-UTC) measurements in order to determine dUT1 with very low latency. The project involves real-time data transfer from Fennoscandia to Japan, near real-time data conversion from MK5 to K5, near real-time correlation with the Japanese software correlator, creating of VLBI databases and quick data analysis. Figure 3 shows the location of the involved sites that form two almost parallel baselines.

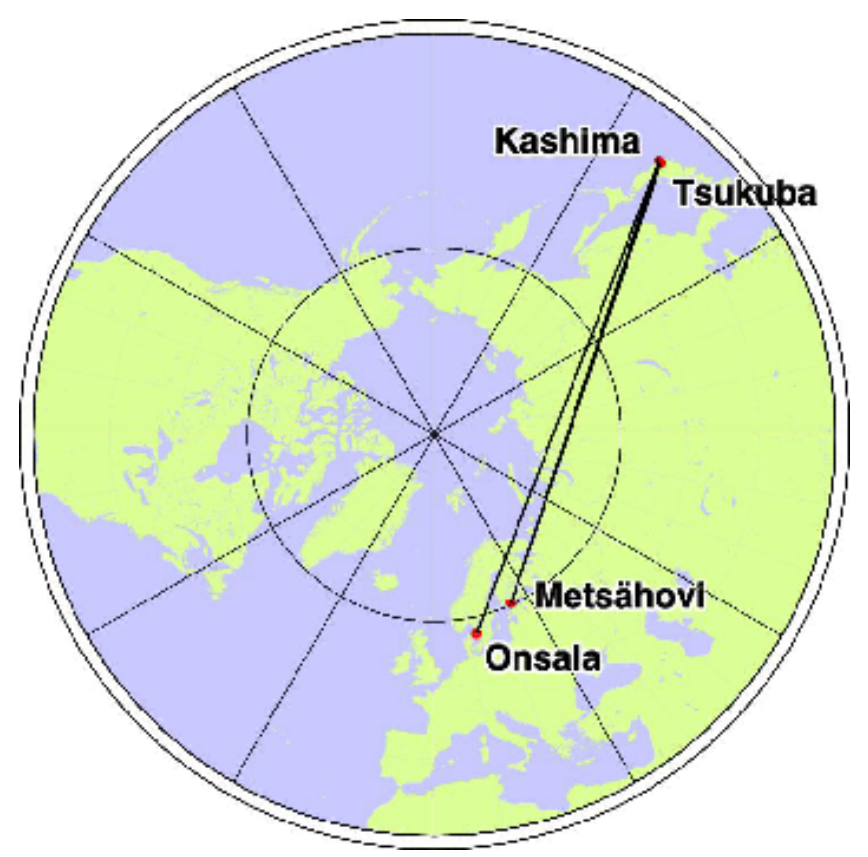

Figure 3: Stations involved in the Fennoscandian-Japanese network for ultra-rapid dUT1 determination

In total we performed 19 such ultra-rapid sessions in 2007. Some used offline e-transfer, some real-time e-VLBI. Data rates between 128 and $512 \mathrm{Mbps}$ were tested. The lowest latency for the final results that we achieved in 2007 was 30 minutes after the end of a session. In 2008 we continued our involvement in the Fennoscandian-Japanese ultra-rapid dUT1 project, together with our colleagues in Metsähovi, Kashima and Tsukuba. The aims for 2008 were to achieve automated telescope operations, real-time data transfer with high data rates, and automated near-real-time correlation and dUT1-analysis. In total we did 27 such sessions during

\footnotetext{
1 “PC EVN Project Plan” http://www.jive.nl/techinfo/evn_docs/evndoc113.pdf
} 
2008. The highlight was the determination of final dUT1-results within 4 minutes after the end of the observing session. During the summer of 2009, first successful experiments were done to do real-time e-VLBI during a complete 24 h VLBI-session. In session R1.385 and RD.0907, where both Onsala and Tsukuba participated, the observational data from Onsala for the baseline Onsala-Tsukuba were successfully sent in real-time to the Tsukuba correlator, converted, correlated and analyzed there during the ongoing $24 \sim \mathrm{h}$ observing session. The success of these efforts is very encouraging and a step towards e-VLBI with distributed correlation. Thus, there are plans to continue the efforts with further experiments during 2009.

\section{Time lab and transfer}

In the spring of 2007 a second maser was installed at the observatory in collaboration with SP Technical Research Institute of Sweden. The aim of the project is to establish a time-lab at Onsala with two masers, a Cesium clock and equipment for GPS-time measurements that contributes to the generation of UTC at BIPM.

In 2008 the time-lab was equipped with an additional Cesium clock, again in collaboration with the SP Technical Research Institute of Sweden. Work is currently ongoing at SP to develop an accurate time-synchronization system using the high-speed optical fibre connection at Onsala. The plan is to install the necessary equipment at Onsala during 2009.

\section{References}

[1] Tudose, V.; Paragi, Z.; Trushkin, S.; Soleri, P.; Fender, R.; Garrett, M.; Spencer, R.; Rushton, A.; Burgess, P.; Kunert-Bajraszewska, E.; Pazderski, E.; Borkowski, K.; Hammargren, R.; Lindqvist, M.; Maccaferri, G, e-VLBI observations of SS 433 in outburst, The Astronomer's Telegram. \#1836. (9 Nov 2008). 